\title{
Common Fixed Point Theorems of Contractions in Partial Cone Metric Spaces over Nonnormal Cones
}

\author{
Zhilong $\mathrm{Li}^{1,2}$ and Shujun Jiang ${ }^{3}$ \\ ${ }^{1}$ School of Statistics, Jiangxi University of Finance and Economics, Nanchang 330013, China \\ ${ }^{2}$ Research Center of Applied Statistics, Jiangxi University of Finance and Economics, Nanchang 330013, China \\ ${ }^{3}$ Department of Mathematics, Jiangxi University of Finance and Economics, Nanchang 330013, China
}

Correspondence should be addressed to Zhilong Li; 1zl771218@sina.com

Received 21 November 2013; Accepted 18 March 2014; Published 10 April 2014

Academic Editor: Sehie Park

Copyright (C) $2014 \mathrm{Z}$. Li and S. Jiang. This is an open access article distributed under the Creative Commons Attribution License, which permits unrestricted use, distribution, and reproduction in any medium, provided the original work is properly cited.

We prove some common fixed point theorems of contractions restricted with variable positive linear bounded mappings in $\theta$-complete partial cone metric spaces over nonnormal cones and present some examples to support the usability of our results.

\section{Introduction}

In 2007, Huang and Zhang [1] introduced cone metric spaces, being unaware that they already existed under the name $K$-metric and $K$-normed spaces that were introduced and used in the middle of the 20th century in [2-9]. In both cases, the set $\mathbb{R}$ of real numbers was replaced by an ordered Banach space $E$. However, Huang and Zhang went further and defined the convergence via interior points of the cone by which the order in $E$ is defined. This approach allows the investigation of cone spaces in the case that the cone is not necessarily normal. Since then, there were many references concerned with fixed point results and common fixed point results in cone metric spaces over a nonnormal cone (see [10-18]). In 2012, based on the definition of cone metric spaces and partial metric spaces introduced by Matthews [19], Sonmez [20, 21] defined a partial cone metric space and proved some fixed point theorems of contractions restricted with constants in complete partial cone metric spaces over normal cones. Recently, without using the normality of the cone, Malhotra et al. [22] and Jiang and Li [23] extended the results of $[20,21]$ to $\theta$-complete partial cone metric spaces. In addition, the contractions considered in [23] are not necessarily restricted with constants but restricted with positive linear bounded mappings.
In this paper, we prove some common fixed point theorems of contractions restricted with variable positive linear bounded mappings in $\theta$-complete partial cone metric spaces over nonnormal cones, which improve the recent results of $[22,23]$.

\section{Preliminaries}

Let $E$ be a topological vector space. A cone of $E$ is a nonempty closed subset $P$ of $E$ such that $a x+b y \in P$ for each $x, y \in P$ and each $a, b \geq 0$, and $P \cap(-P)=\{\theta\}$, where $\theta$ is the zero element of $E$. A cone $P$ of $E$ determines a partial order $\preceq$ on $E$ by $x \preceq y \Leftrightarrow y-x \in P$ for each $x, y \in X$. In this case, $E$ is called an ordered topological vector space.

A cone $P$ of a topological vector space $E$ is solid if int $P \neq \varnothing$, where int $P$ is the interior of $P$. For each $x, y \in E$ with $y-x \in \operatorname{int} P$, we write $x \ll y$. Let $P$ be a solid cone of a topological vector space $E$. A sequence $\left\{u_{n}\right\}$ of $E$ weakly converges [22] to $u \in E$ (denote $u_{n} \stackrel{w}{\rightarrow} u$ ) if, for each $\epsilon \in \operatorname{int} P$, there exists a positive integer $n_{0}$ such that $u-\epsilon \ll u_{n} \ll u+\epsilon$ for all $n \geq n_{0}$.

A subset $D$ of a topological vector space $E$ is order-convex if $[x, y] \subset D$ for each $x, y \in D$ with $x \preceq y$, where $[x, y]=$ $\{z \in E: x \preceq z \preceq y\}$. An ordered topological vector space $E$ is 
order-convex if it has a base of neighborhoods of $\theta$ consisting of order-convex subsets. In this case, the cone $P$ is said to be normal. In the case of a normed vector space, this condition means that the unit ball is order-convex, which is equivalent to the condition that there is some positive number $N$ such that $x, y \in E$ and $\theta \preceq x \preceq y$ implies that $\|x\| \leq N\|y\|$, and the minimal $N$ is called a normal constant of $P$. Another equivalent condition is that

$$
\inf \{\|x+y\|: x, y \in P,\|x\|=\|y\|=1\}>0 .
$$

It is not hard to conclude from (1) that $P$ is a nonnormal cone of a normed vector space $(E,\|\cdot\|)$ if and only if there exist sequences $\left\{u_{n}\right\},\left\{v_{n}\right\} \subset P$ such that

$$
u_{n}+v_{n} \stackrel{\|\cdot\|}{\longrightarrow} \theta \nRightarrow u_{n} \stackrel{\|\cdot\|}{\longrightarrow} \theta,
$$

which implies that the Sandwich theorem does not hold. However, the Sandwich theorem holds in the sense of weak convergence even if $P$ is a nonnormal cone.

Lemma 1 (Sandwich theorem). Let $P$ be a solid cone of a topological vector space $E$ and $\left\{u_{n}\right\},\left\{v_{n}\right\},\left\{w_{n}\right\} \subset$ E. If

$$
u_{n} \preceq w_{n} \preceq v_{n}, \quad \forall n,
$$

and there exists some $w \in E$ such that $u_{n} \stackrel{w}{\rightarrow} w$ and $v_{n} \stackrel{w}{\rightarrow} w$, then $w_{n} \stackrel{w}{\rightarrow} w$.

Proof. By $u_{n} \stackrel{w}{\rightarrow} w$ and $v_{n} \stackrel{w}{\rightarrow} w$, for each $\epsilon \in$ int $P$, there exists some positive integer $n_{0}$ such that, for all $n \geq n_{0}$,

$$
w-\epsilon \ll u_{n}, \quad v_{n} \ll w+\epsilon .
$$

Thus, by (3) and (4), we have $w-\epsilon \ll u_{n} \preceq w_{n} \preceq v_{n} \preceq$ $v_{n} \ll w+\epsilon$ for all $n \geq n_{0}$; that is, $w_{n} \stackrel{w}{\rightarrow} w$. The proof is completed.

The following lemma is needed in further arguments, which directly follows from Lemma 1 and Remark 1 of [23].

Lemma 2. Let $P$ be a solid cone of a normed vector space $(E,\|\cdot\|)$. Then, for each sequence $\left\{u_{n}\right\} \subset E, u_{n} \stackrel{\|\cdot\|}{\longrightarrow} u$ implies $u_{n} \stackrel{w}{\rightarrow} u$. Moreover, if $P$ is normal, then $u_{n} \stackrel{w}{\rightarrow} u$ implies $u_{n} \stackrel{\|\cdot\|}{\longrightarrow} u$.

Let $P$ be a cone of a normed vector space $(E,\|\cdot\|)$ and $L$ : $E \rightarrow E$. The mapping $L$ is said to be a positive linear bounded mapping if $L(P) \subset P, L(u+v)=L u+L v$ for each $u, v \in E$, and there exists some positive real number $M>0$ such that $\|L\| \leq M$. In the sequel, $\mathfrak{L}$ and $I$ will denote the family of all positive linear bounded mappings and the identity mapping, respectively.

Lemma 3. Let $P$ be a solid cone of a normed vector space $(E,\|\cdot\|),\left\{K_{n}\right\} \subset \mathfrak{Q}$ and $\left\{u_{n}\right\} \subset P$. If $u_{n} \stackrel{w}{\rightarrow} \theta$ and $\sup _{n}\left\|K_{n}\right\|<$ $+\infty$, then $K_{n} u_{n} \stackrel{w}{\rightarrow} \theta$.
Proof. Let $\widetilde{K}_{n}=b K_{n}$, for all $n$, where

$$
b= \begin{cases}1, & \sup _{n}\left\|K_{n}\right\|<1, \\ \frac{1}{\sup _{n}\left\|K_{n}\right\|+1}, & \sup _{n}\left\|K_{n}\right\| \geq 1 .\end{cases}
$$

It is clear that $\left\|\widetilde{K}_{n}\right\| \leq b\left\|K_{n}\right\|<1$ for all $n$, and hence, for all $n$, the inverse of $I-\widetilde{K}_{n}$ exists (denoted by $\left.\left(I-\widetilde{K}_{n}\right)^{-1}\right)$. It follows from $\left\{K_{n}\right\} \subset \mathbb{Q}$ that for all $n\left(I-\widetilde{K}_{n}\right)^{-1} \in \mathbb{Q}$ for all $n$, and then $I-\widetilde{K}_{n} \in \mathfrak{Q}$ for all $n$. By Lemma 2 and $u_{n} \stackrel{w}{\rightarrow} \theta$, for each $\epsilon \in \operatorname{int} P$, there exists some positive integer $n_{0}$ such that $\theta \preceq$ $u_{n} \ll b \epsilon$ for all $n \geq n_{0}$. Note that $I-\widetilde{K}_{n} \in \mathfrak{Q}$ for all $n$ implies that $\widetilde{K}_{n} u \preceq u$ for all $n$ and each $u \in P$; then, $\theta \preceq K_{n} u_{n}=$ $\widetilde{K}_{n}\left(u_{n} / b\right) \preceq\left(u_{n} / b\right) \ll \epsilon$ for all $n \geq n_{0}$; that is, $K_{n} u_{n} \stackrel{w}{\rightarrow} \theta$. The proof is completed.

Let $X$ be a nonempty set and let $P$ be a cone of a topological vector space $E$. A partial cone metric on $X$ is a mapping $p: X \times X \rightarrow P$ such that, for each $x, y, z \in X$,

$$
\begin{aligned}
& \text { (p1) } p(x, y)=p(x, x)=p(y, y) \Leftrightarrow x=y \text {; } \\
& \text { (p2) } p(x, y)=p(y, x) ; \\
& \text { (p3) } p(x, x) \leq p(x, y) ; \\
& \text { (p4) } p(x, y) \leq p(x, z)+p(z, y)-p(z, z) .
\end{aligned}
$$

The pair $(X, p)$ is called a partial cone metric space over $P$. A partial cone metric $p$ on $X$ over a solid cone $P$ generates a topology $\tau_{p}$ on $X$ which has a base of the family of open $p$ balls $\left\{B_{p}(x, \epsilon): x \in X, \theta \ll \epsilon\right\}$, where $B_{p}(x, \epsilon)=\{y \in X$ : $p(x, y) \ll p(x, x)+\epsilon\}$ for each $x \in X$ and each $\epsilon \in \operatorname{int} P$.

Let $(X, p)$ be a partial cone metric space over a solid cone $P$ of a topological vector space $E$. A sequence $\left\{x_{n}\right\}$ of $X$ converges to $x \in X$ (denoted by $x_{n} \stackrel{\tau_{p}}{\longrightarrow} x$ ) if $p\left(x_{n}, x\right) \stackrel{w}{\rightarrow}$ $p(x, x)$. A sequence $\left\{x_{n}\right\}$ of $X$ is $\theta$-Cauchy, if $p\left(x_{n}, x_{m}\right) \stackrel{w}{\rightarrow} \theta$. The partial cone metric space $(X, p)$ is $\theta$-complete, if each $\theta$ Cauchy sequence $\left\{x_{n}\right\}$ of $X$ converges to a point $x \in X$ such that $p(x, x)=\theta$. Every complete partial cone metric space $(X, p)$ is $\theta$-complete, but the converse may not be true (see [23]).

\section{Common Fixed Point Theorems}

Let $(X, p)$ be a partial cone metric space. The mappings $T, S$ : $X \rightarrow X$ are called contractions restricted with variable positive linear bounded mappings if there exist $L_{i}: X \times X \rightarrow$ $\mathcal{L}(i=1,2,3,4)$ such that

$$
\begin{aligned}
p(T x, S y) \leq & L_{1}(x, y) p(x, y) \\
+ & L_{2}(x, y) p(x, T x)+L_{3}(x, y) p(y, S y) \\
+ & L_{4}(x, y)[p(x, S y)+p(y, T x)], \\
& \forall x, y \in X .
\end{aligned}
$$

In particular, if (6) holds with $L_{i}(x, y) \equiv A_{i}(i=1,2,3,4)$ and $A_{i} \in \mathfrak{L}(i=1,2,3,4)$, then $T$ and $S$ are called contractions restricted with positive linear bounded mappings. 
We first present a common fixed point theorem of contractions restricted with variable positive linear bounded mappings in a partial cone metric space over a nonnormal cone. In the sequel, $\mathbb{N}$ will denote the set of all nonnegative integer numbers.

Theorem 4. Let $(X, p)$ be a $\theta$-complete partial cone metric space over a solid cone $P$ of a normed vector space $(E,\|\cdot\|)$, and let $T, S: X \rightarrow X$ be contractions restricted with variable positive linear bounded mappings. If

$$
\begin{array}{r}
\rho\left(L_{3}(x, y)+L_{4}(x, y)\right)<1, \quad \rho\left(L_{2}(x, y)+L_{4}(x, y)\right)<1, \\
\forall x, y \in X,
\end{array}
$$

$l_{1} l_{2}<1$ and $l_{3}<+\infty$, where $\rho(\cdot)$ denotes the spectral radius of linear bounded mappings,

$$
\begin{gathered}
l_{1}=\sup _{x, y \in X}\left\|K_{1}(x, y)\right\|, \\
l_{2}=\sup _{x, y \in X}\left\|K_{2}(x, y)\right\|, \\
l_{3}=\sup _{x, y \in X}\left\|K_{3}(x, y)\right\|, \\
K_{1}(x, y)=\widetilde{L}_{1}(x, y)\left[L_{1}(x, y)+L_{2}(x, y)+L_{4}(x, y)\right], \\
K_{2}(x, y)=\widetilde{L}_{2}(x, y)\left[L_{1}(x, y)+L_{3}(x, y)+L_{4}(x, y)\right], \\
K_{3}(x, y)=\widetilde{L}_{2}(x, y)\left[I+L_{3}(x, y)+L_{4}(x, y)\right], \\
\forall x, y \in X,
\end{gathered}
$$

where $\widetilde{L}_{1}(x, y)$ and $\widetilde{L}_{2}(x, y)$ denote the inverses of $I-L_{3}(x, y)-$ $L_{4}(x, y)$ and $I-L_{2}(x, y)-L_{4}(x, y)$, respectively. Then, $T$ and $S$ have a common fixed point in $X$. Moreover, if

$$
\begin{array}{r}
\rho\left(L_{1}(x, y)+L_{2}(x, y)+L_{3}(x, y)+2 L_{4}(x, y)\right)<1, \\
\forall x, y \in X,
\end{array}
$$

then $T$ and $S$ have a unique common fixed point $x^{*} \in X$ such that, for each $x_{0} \in X, x_{n} \stackrel{\tau_{p}}{\longrightarrow} x^{*}$, where $x_{n}$ is defined by

$$
x_{n+1}= \begin{cases}T x_{n}, & n \text { is an even number, } \\ S x_{n}, & n \text { is an odd number. }\end{cases}
$$

Proof. For each $x, y \in X$, by (7), the inverses of $I-L_{3}(x, y)-$ $L_{4}(x, y)$ and $I-L_{2}(x, y)-L_{4}(x, y)$ exist. Then, it is clear that $\widetilde{L}_{1}$ and $\widetilde{L}_{2}$ are meaningful, and so $K_{1}, K_{2}, K_{3}$ are well defined. Moreover, by Neumann's formula,

$$
\begin{aligned}
& \widetilde{L}_{1}(x, y)=\sum_{i=0}^{\infty}\left[L_{3}(x, y)+L_{4}(x, y)\right]^{i}, \\
& \widetilde{L}_{2}(x, y)=\sum_{i=0}^{\infty}\left[L_{2}(x, y)+L_{4}(x, y)\right]^{i},
\end{aligned}
$$

which together with $L_{i}: X \times X \rightarrow \mathfrak{L}(i=2,3,4)$ implies that $\widetilde{L}_{i}: X \times X \rightarrow \mathfrak{L}(i=1,2)$, and hence $K_{i}: X \times X \rightarrow$ $\mathfrak{L}(i=1,2,3)$. By (6), (11), (p4), and $L_{4}: X \times X \rightarrow \mathfrak{L}$,

$$
\begin{aligned}
& p\left(x_{2 k+1}, x_{2 k+2}\right)= p\left(T x_{2 k}, S x_{2 k+1}\right) \\
& \leq L_{1}\left(x_{2 k}, x_{2 k+1}\right) p\left(x_{2 k}, x_{2 k+1}\right) \\
&+L_{2}\left(x_{2 k}, x_{2 k+1}\right) p\left(x_{2 k}, x_{2 k+1}\right) \\
&+L_{3}\left(x_{2 k}, x_{2 k+1}\right) p\left(x_{2 k+1}, x_{2 k+2}\right) \\
&+L_{4}\left(x_{2 k}, x_{2 k+1}\right)\left[p\left(x_{2 k}, x_{2 k+2}\right)\right. \\
&\left.+p\left(x_{2 k+1}, x_{2 k+1}\right)\right] \\
& \leq L_{1}\left(x_{2 k}, x_{2 k+1}\right) p\left(x_{2 k}, x_{2 k+1}\right) \\
&+L_{2}\left(x_{2 k}, x_{2 k+1}\right) p\left(x_{2 k}, x_{2 k+1}\right) \\
&+L_{3}\left(x_{2 k}, x_{2 k+1}\right) p\left(x_{2 k+1}, x_{2 k+2}\right) \\
&+L_{4}\left(x_{2 k}, x_{2 k+1}\right)\left[p\left(x_{2 k}, x_{2 k+1}\right)\right. \\
&\left.+p\left(x_{2 k+1}, x_{2 k+2}\right)\right], \\
& \forall k \in \mathbb{N},
\end{aligned}
$$

and so

$$
\begin{aligned}
{[I-} & \left.L_{3}\left(x_{2 k}, x_{2 k+1}\right)-L_{4}\left(x_{2 k}, x_{2 k+1}\right)\right] p\left(x_{2 k+1}, x_{2 k+2}\right) \\
\leq & {\left[L_{1}\left(x_{2 k}, x_{2 k+1}\right)+L_{2}\left(x_{2 k}, x_{2 k+1}\right)\right.} \\
& \left.+L_{4}\left(x_{2 k}, x_{2 k+1}\right)\right] p\left(x_{2 k}, x_{2 k+1}\right), \quad \forall k \in \mathbb{N} .
\end{aligned}
$$

Act the above inequality with $\widetilde{L}_{1}\left(x_{2 k}, x_{2 k+1}\right)$; then, by $\widetilde{L}_{1}: X \times$ $X \rightarrow \mathfrak{Q}$,

$$
p\left(x_{2 k+1}, x_{2 k+2}\right) \leq K_{1}\left(x_{2 k}, x_{2 k+1}\right) p\left(x_{2 k}, x_{2 k+1}\right), \quad \forall k \in \mathbb{N} .
$$

Similarly, by (6), (p3), (p4), and $L_{4}: X \times X \rightarrow \mathfrak{Q}$,

$$
\begin{aligned}
p\left(x_{2 k+2}, x_{2 k+3}\right)= & p\left(x_{2 k+3}, x_{2 k+2}\right) \\
= & p\left(T x_{2 k+2}, S x_{2 k+1}\right) \\
\leq & L_{1}\left(x_{2 k+2}, x_{2 k+1}\right) p\left(x_{2 k+2}, x_{2 k+1}\right) \\
& +L_{2}\left(x_{2 k+2}, x_{2 k+1}\right) p\left(x_{2 k+2}, x_{2 k+3}\right) \\
& +L_{3}\left(x_{2 k+2}, x_{2 k+1}\right) p\left(x_{2 k+1}, x_{2 k+2}\right) \\
& +L_{4}\left(x_{2 k+2}, x_{2 k+1}\right)\left[p\left(x_{2 k+2}, x_{2 k+2}\right)\right. \\
& \left.\quad+p\left(x_{2 k+1}, x_{2 k+3}\right)\right] \\
\leq & L_{1}\left(x_{2 k+2}, x_{2 k+1}\right) p\left(x_{2 k+2}, x_{2 k+1}\right) \\
& +L_{2}\left(x_{2 k+2}, x_{2 k+1}\right) p\left(x_{2 k+2}, x_{2 k+3}\right) \\
& +L_{3}\left(x_{2 k+2}, x_{2 k+1}\right) p\left(x_{2 k+1}, x_{2 k+2}\right)
\end{aligned}
$$




$$
\begin{aligned}
+L_{4}\left(x_{2 k+2}, x_{2 k+1}\right)[ & p\left(x_{2 k+2}, x_{2 k+1}\right) \\
+ & \left.p\left(x_{2 k+2}, x_{2 k+3}\right)\right],
\end{aligned}
$$

$\forall k \in \mathbb{N}$,

and so

$$
\begin{aligned}
{[I-} & \left.L_{2}\left(x_{2 k+2}, x_{2 k+1}\right)-L_{4}\left(x_{2 k+2}, x_{2 k+1}\right)\right] p\left(x_{2 k+2}, x_{2 k+3}\right) \\
\leq & {\left[L_{1}\left(x_{2 k+2}, x_{2 k+1}\right)+L_{3}\left(x_{2 k+2}, x_{2 k+1}\right)\right.} \\
& \left.+L_{4}\left(x_{2 k+2}, x_{2 k+1}\right)\right] p\left(x_{2 k+2}, x_{2 k+1}\right), \quad \forall k \in \mathbb{N} .
\end{aligned}
$$

Act the above inequality with $\widetilde{L}_{2}\left({ }_{2 k+2}, x_{2 k+1}\right)$; then, by $\widetilde{L}_{2}$ : $X \times X \rightarrow \mathbf{Q}$,

$$
\begin{array}{r}
p\left(x_{2 k+2}, x_{2 k+3}\right) \preceq K_{2}\left(x_{2 k+2}, x_{2 k+1}\right) p\left(x_{2 k+1}, x_{2 k+2}\right), \\
\forall k \in \mathbb{N} .
\end{array}
$$

Moreover, by (15), (18), and $K_{1}, K_{2}: X \times X \rightarrow \mathfrak{Q}$,

$$
\begin{aligned}
p\left(x_{2 k+1}, x_{2 k+2}\right) \leq & K_{1}\left(x_{2 k}, x_{2 k+1}\right) \\
& \times K_{2}\left(x_{2 k}, x_{2 k-1}\right) \cdots K_{1}\left(x_{0}, x_{1}\right) p\left(x_{0}, x_{1}\right), \\
p\left(x_{2 k+2}, x_{2 k+3}\right) \leq & K_{2}\left(x_{2 k+2}, x_{2 k+1}\right) K_{1}\left(x_{2 k}, x_{2 k+1}\right) \\
& \times K_{2}\left(x_{2 k}, x_{2 k-1}\right) \cdots K_{1}\left(x_{0}, x_{1}\right) p\left(x_{0}, x_{1}\right),
\end{aligned}
$$$$
\forall k \in \mathbb{N} \text {. }
$$

In the following, we will prove that

$$
p\left(x_{n}, x_{m}\right) \stackrel{w}{\rightarrow} \theta .
$$

For all $m>n$, we have four cases: (i) $m=2 p+1, n=2 q+1$; (ii) $m=2 p+1, n=2 q$; (iii) $m=2 p, n=2 q+1$; and (iv) $m=2 p, n=2 q$, where $p$ and $q$ are two nonnegative integers such that $p>q$. We only show that (20) holds for case (i); the proofs of the other three cases are similar.

It follows from (p4) and (19) that

$$
\begin{aligned}
\theta \preceq & p\left(x_{n}, x_{m}\right) \\
= & p\left(x_{2 q+1}, x_{2 p+1}\right) \\
\leq & p\left(x_{2 q+1}, x_{2 q+2}\right)+p\left(x_{2 q+2}, x_{2 q+3}\right) \\
& +\cdots+p\left(x_{2 p-1}, x_{2 p}\right)+p\left(x_{2 p}, x_{2 p+1}\right) \\
\leq & p_{K_{1} K_{2}}\left(x_{0}, x_{1}\right) \\
= & K_{1}\left(x_{2 q}, x_{2 q+1}\right) \\
& \times K_{2}\left(x_{2 q}, x_{2 q-1}\right) \cdots K_{1}\left(x_{0}, x_{1}\right) p\left(x_{0}, x_{1}\right) \\
& +K_{2}\left(x_{2 q+2}, x_{2 q+1}\right) K_{1}\left(x_{2 q}, x_{2 q+1}\right)
\end{aligned}
$$

$$
\begin{aligned}
& \times K_{2}\left(x_{2 q}, x_{2 q-1}\right) \cdots K_{1}\left(x_{0}, x_{1}\right) p\left(x_{0}, x_{1}\right) \\
& +\cdots+K_{1}\left(x_{2 p-2}, x_{2 p-1}\right) \\
& \times K_{2}\left(x_{2 p-2}, x_{2 p-3}\right) \cdots K_{1}\left(x_{0}, x_{1}\right) p\left(x_{0}, x_{1}\right) \\
& +K_{2}\left(x_{2 p}, x_{2 p-1}\right) \\
& \times K_{1}\left(x_{2 p-2}, x_{2 p-1}\right) \cdots K_{1}\left(x_{0}, x_{1}\right) p\left(x_{0}, x_{1}\right), \\
& \forall p>q .
\end{aligned}
$$

By $l_{1} l_{2}<1$,

$$
\begin{aligned}
& \left\|p_{K_{1} K_{2}}\left(x_{0}, x_{1}\right)\right\| \\
& \leq\left(l_{1}^{q+1} l_{2}^{q}+l_{1}^{q+1} l_{2}^{q+1}+\cdots+l_{1}^{p+1} l_{2}^{p}+l_{1}^{p} l_{2}^{p}\right)\left\|p\left(x_{0}, x_{1}\right)\right\| \\
& \quad=\left(l_{1} \sum_{i=q}^{p}\left(l_{1} l_{2}\right)^{i}+\sum_{i=q+1}^{p}\left(l_{1} l_{2}\right)^{i}\right)\left\|p\left(x_{0}, x_{1}\right)\right\| \\
& \leq \frac{\left(l_{1}+l_{1} l_{2}\right)\left(l_{1} l_{2}\right)^{q}\left\|p\left(x_{0}, x_{1}\right)\right\|}{1-l_{1} l_{2}}, \quad \forall p>q,
\end{aligned}
$$

which implies that $p_{K_{1} K_{2}}\left(x_{0}, x_{1}\right) \stackrel{\|\cdot\|}{\longrightarrow} \theta$, and hence $p_{K_{1} K_{2}}\left(x_{0}\right.$, $\left.x_{1}\right) \stackrel{w}{\rightarrow} \theta$ by Lemma 2 . Thus, by (21) and Lemma $1, p\left(x_{n}\right.$, $\left.x_{m}\right) \stackrel{w}{\rightarrow} \theta$; that is, $(20)$ holds. It is proved that $\left\{x_{n}\right\}$ is a $\theta$ Cauchy sequence in $(X, p)$, and so by the $\theta$-completeness of $(X, p)$, there exists $x^{*} \in X$ such that $x_{n} \stackrel{\tau_{p}}{\longrightarrow} x^{*}$ and $p\left(x^{*}\right.$, $\left.x^{*}\right)=\theta$; that is,

$$
p\left(x_{n}, x^{*}\right) \stackrel{w}{\rightarrow} \theta
$$

For all $k \in \mathbb{N}$, by $(6)$ and (p4),

$$
\begin{aligned}
p\left(T x^{*}, x^{*}\right) \preceq & p\left(T x^{*}, x_{2 k}\right)+p\left(x_{2 k}, x^{*}\right) \\
= & p\left(T x^{*}, S x_{2 k-1}\right)+p\left(x_{2 k}, x^{*}\right) \\
\preceq & L_{1}\left(x^{*}, x_{2 k-1}\right) p\left(x^{*}, x_{2 k-1}\right) \\
& +L_{2}\left(x^{*}, x_{2 k-1}\right) p\left(x^{*}, T x^{*}\right) \\
& +L_{3}\left(x^{*}, x_{2 k-1}\right) p\left(x_{2 k-1}, x_{2 k}\right) \\
& +L_{4}\left(x^{*}, x_{2 k-1}\right) \\
& \times\left[p\left(x^{*}, x_{2 k}\right)+p\left(x_{2 k-1}, T x^{*}\right)\right] \\
& +p\left(x_{2 k}, x^{*}\right)
\end{aligned}
$$




$$
\begin{aligned}
\preceq & L_{1}\left(x^{*}, x_{2 k-1}\right) p\left(x^{*}, x_{2 k-1}\right) \\
+ & L_{2}\left(x^{*}, x_{2 k-1}\right) p\left(x^{*}, T x^{*}\right) \\
+ & L_{3}\left(x^{*}, x_{2 k-1}\right) \\
\times & {\left[p\left(x_{2 k-1}, x^{*}\right)+p\left(x^{*}, x_{2 k}\right)\right] } \\
+ & L_{4}\left(x^{*}, x_{2 k-1}\right) \\
\times & {\left[p\left(x^{*}, x_{2 k}\right)+p\left(x_{2 k-1}, x^{*}\right)\right.} \\
& \left.+p\left(x^{*}, T x^{*}\right)\right]+p\left(x_{2 k}, x^{*}\right),
\end{aligned}
$$

and so

$$
\begin{aligned}
& {\left[I-L_{2}\left(x^{*}, x_{2 k-1}\right)-L_{4}\left(x^{*}, x_{2 k-1}\right)\right] p\left(T x^{*}, x^{*}\right)} \\
& \preceq\left[L_{1}\left(x^{*}, x_{2 k-1}\right)+L_{3}\left(x^{*}, x_{2 k-1}\right)\right. \\
& \left.\quad+L_{4}\left(x^{*}, x_{2 k-1}\right)\right] p\left(x^{*}, x_{2 k-1}\right) \\
& \quad+\left[I+L_{3}\left(x^{*}, x_{2 k-1}\right)+L_{4}\left(x^{*}, x_{2 k-1}\right)\right] p\left(x_{2 k}, x^{*}\right) .
\end{aligned}
$$

Act the above inequality with $\widetilde{L}_{2}\left(x^{*}, x_{2 k-1}\right)$; then, by $\widetilde{L}_{2}: X \times$ $X \rightarrow \mathfrak{R}$,

$$
\begin{aligned}
\theta & \leq p\left(T x^{*}, x^{*}\right) \\
& \preceq K_{2,2 k-1} p\left(x^{*}, x_{2 k-1}\right)+K_{3,2 k-1} p\left(x_{2 k}, x^{*}\right), \quad \forall k \in \mathbb{N},
\end{aligned}
$$

where $K_{2,2 k-1}=K_{2}\left(x^{*}, x_{2 k-1}\right)$ and $K_{3,2 k-1}=K_{3}\left(x^{*}, x_{2 k-1}\right)$. It is clear that $\left\{K_{2,2 k-1}\right\},\left\{K_{3,2 k-1}\right\} \subset \mathbf{Q}$ and $\sup _{k}\left\|K_{3,2 k-1}\right\|<$ $+\infty, \sup _{k}\left\|K_{3,2 k-1}\right\|<+\infty$ by $l_{1} l_{2}<1$ and $l_{3}<+\infty$. Then, it follows from Lemma 3 and (23) that

$$
K_{2,2 k-1} p\left(x^{*}, x_{2 k-1}\right)+K_{3,2 k-1} p\left(x_{2 k}, x^{*}\right) \stackrel{w}{\rightarrow} \theta,
$$

which together with Lemma 1 and (26) implies that $p\left(T x^{*}\right.$, $\left.x^{*}\right)=\theta$. Therefore, $T x^{*}=x^{*}$ by (p1) and (p3). Similarly, we can show that $S x^{*}=x^{*}$. Hence, $x^{*}$ is a common fixed point of $T$ and $S$.

Now, we show the uniqueness of fixed point. Let $x$ and $x^{*}$ be two common fixed points of $T$ and $S$. Then, by (6), (p3), and $L_{i}: X \times X \rightarrow \mathfrak{Q}(i=2,3)$,

$$
\begin{aligned}
p\left(x^{*}, x\right)= & p\left(T x^{*}, S x\right) \\
\leq & L_{1}\left(x^{*}, x\right) p\left(x^{*}, x\right)+L_{2}\left(x^{*}, x\right) p\left(x^{*}, T x^{*}\right) \\
& +L_{3}\left(x^{*}, x\right) p(x, S x) \\
& +L_{4}\left(x^{*}, x\right)\left[p\left(x^{*}, S x\right)+p\left(x, T x^{*}\right)\right] \\
= & {\left[L_{1}\left(x^{*}, x\right)+2 L_{4}\left(x^{*}, x\right)\right] p\left(x^{*}, x\right) } \\
& +L_{2}\left(x^{*}, x\right) p\left(x^{*}, x^{*}\right)+L_{3}\left(x^{*}, x\right) p(x, x) \\
\preceq & {\left[L_{1}\left(x^{*}, x\right)+L_{2}\left(x^{*}, x\right)\right.} \\
& \left.+L_{3}\left(x^{*}, x\right)+2 L_{4}\left(x^{*}, x\right)\right] p\left(x^{*}, x\right),
\end{aligned}
$$

and so

$$
\begin{aligned}
& {\left[I-L_{1}\left(x^{*}, x\right)-L_{2}\left(x^{*}, x\right)-L_{3}\left(x^{*}, x\right)\right.} \\
& \left.-2 L_{4}\left(x^{*}, x\right)\right] p\left(x^{*}, x\right) \preceq \theta .
\end{aligned}
$$

It follows from (9) that the inverse of $I-L_{1}\left(x^{*}, x\right)-$ $L_{2}\left(x^{*}, x\right)-L_{3}\left(x^{*}, x\right)-2 L_{4}\left(x^{*}, x\right)$ exists (denoted by $\left.\left[I-L_{1}\left(x^{*}, x\right)-L_{2}\left(x^{*}, x\right)-L_{3}\left(x^{*}, x\right)-2 L_{4}\left(x^{*}, x\right)\right]^{-1}\right)$, and $\left[I-L_{1}\left(x^{*}, x\right)-L_{2}\left(x^{*}, x\right)-L_{3}\left(x^{*}, x\right)-2 L_{4}\left(x^{*}, x\right)\right]^{-1} \in \mathbf{\Omega}$ by Neumann's formula. Act (29) with $\left[I-L_{1}\left(x^{*}\right.\right.$, $\left.x)-L_{2}\left(x^{*}, x\right)-L_{3}\left(x^{*}, x\right)-2 L_{4}\left(x^{*}, x\right)\right]^{-1}$; then, $p\left(x^{*}, x\right) \preceq$ $\theta$, and hence $x=x^{*}$ by (p1) and (p3). The proof is completed.

Remark 5. Theorem 3 of [23] is a special case of Theorem 4 with $T=S$ and $L_{i}(x, y) \equiv c_{i} I(i=1,2,3,4)$, where $c_{i}(i=$ $1,2,3,4)$ are nonnegative numbers such that $c_{1}+c_{2}+c_{3}+2 c_{4}<$ 1.

Note that Theorem 4 is still valid if $L_{i}(i=1,2,3,4)$ are replaced with nonnegative bounded real functions; then, we have the following corollary for which $E$ is not necessarily confined to a normed vector space.

Corollary 6. Let $(X, p)$ be a $\theta$-complete partial cone metric space over a solid cone $P$ of a topological vector space $E$ and $T, S: X \rightarrow X$. Assume that there exist four nonnegative bounded functions $\alpha_{i}: X \times X \rightarrow[0,+\infty)(i=1,2,3,4)$ such that

$$
\begin{aligned}
p(T x, S y) \preceq & \alpha_{1}(x, y) p(x, y) \\
+ & \alpha_{2}(x, y) p(x, T x)+\alpha_{3}(x, y) p(y, S y) \\
+ & \alpha_{4}(x, y)[p(x, S y)+p(y, T x)], \\
& \forall x, y \in X .
\end{aligned}
$$

If

$$
\begin{array}{r}
\alpha_{1}(x, y)+\alpha_{2}(x, y)+\alpha_{3}(x, y)+2 \alpha_{4}(x, y)<1, \\
\forall x, y \in X,
\end{array}
$$

$m_{1} m_{2}<1$ and $m_{3}<+\infty$, where

$$
\begin{aligned}
& m_{1}=\sup _{x, y \in X} \frac{\alpha_{1}(x, y)+\alpha_{2}(x, y)+\alpha_{4}(x, y)}{1-\alpha_{3}(x, y)-\alpha_{4}(x, y)}, \\
& m_{2}=\sup _{x, y \in X} \frac{\alpha_{1}(x, y)+\alpha_{3}(x, y)+\alpha_{4}(x, y)}{1-\alpha_{2}(x, y)-\alpha_{4}(x, y)}, \\
& m_{3}=\sup _{x, y \in X} \frac{1+\alpha_{3}(x, y)+\alpha_{4}(x, y)}{1-\alpha_{2}(x, y)-\alpha_{4}(x, y)} .
\end{aligned}
$$

Then, $T$ and S have a unique common fixed point $x^{*} \in X$ such that, for each $x_{0} \in X, x_{n} \stackrel{\tau_{p}}{\rightarrow} x^{*}$, where $x_{n}$ is defined by (11).

Corollary 7. Let $(X, p)$ be a $\theta$-complete partial cone metric space over a solid cone $P$ of a normed vector space $(E,\|\cdot\|)$, and 
let $T, S: X \rightarrow X$ be contractions restricted with positive linear bounded mappings. If

$$
\begin{aligned}
& \left\|A_{1}+A_{2}+A_{4}\right\|+\left\|A_{3}+A_{4}\right\|<1, \\
& \left\|A_{1}+A_{3}+A_{4}\right\|+\left\|A_{2}+A_{4}\right\|<1,
\end{aligned}
$$

then $T$ and $S$ have a unique common fixed point $x^{*} \in X$ such that, for each $x_{0} \in X, x_{n} \stackrel{\tau_{p}}{\rightarrow} x^{*}$, where $x_{n}$ is defined by (11).

Proof. Let $L_{i}(x, y) \equiv A_{i}(i=1,2,3,4)$. It is easy to check that (6) holds with $L_{i}(x, y) \equiv A_{i}(i=1,2,3,4), L_{i}, K_{i}: X \times X \rightarrow$ $\mathfrak{Q}(i=1,2,3,4)$, where $K_{1}(x, y) \equiv\left(I-A_{3}-A_{4}\right)^{-1}\left(A_{1}+\right.$ $\left.A_{2}+A_{4}\right), K_{2}(x, y) \equiv\left(I-A_{2}-A_{4}\right)^{-1}\left(A_{1}+A_{3}+A_{4}\right)$, and $K_{3}(x, y) \equiv\left(I-A_{2}-A_{4}\right)^{-1}\left(I+A_{3}+A_{4}\right)$. By (33) and Neumann's formula,

$$
\begin{aligned}
\left\|K_{1}(x, y)\right\| & \leq\left\|\left(I-A_{3}-A_{4}\right)^{-1}\right\|\left\|A_{1}+A_{2}+A_{4}\right\| \\
& \leq \frac{\left\|A_{1}+A_{2}+A_{4}\right\|}{1-\left\|A_{3}+A_{4}\right\|}<1, \\
\left\|K_{2}(x, y)\right\| & \leq\left\|\left(I-A_{2}-A_{4}\right)^{-1}\right\|\left\|A_{1}+A_{3}+A_{4}\right\| \\
& \leq \frac{\left\|A_{1}+A_{3}+A_{4}\right\|}{1-\left\|A_{2}+A_{4}\right\|}<1, \\
\left\|K_{3}(x, y)\right\| & \leq\left\|\left(I-A_{2}-A_{4}\right)^{-1}\right\|\left\|I+A_{3}+A_{4}\right\| \\
& \leq \frac{2}{1-\left\|A_{2}+A_{4}\right\|}<+\infty,
\end{aligned}
$$

for each $x, y \in X$; that is, $l_{1} l_{2}<1$ and $l_{3}<+\infty$. Note that both (7) and (10) hold with $L_{i}(x, y) \equiv A_{i}(i=1,2,3,4)$ by (33); then, the conclusion directly follows from Theorem 4. The proof is completed.

Note that (33) hold naturally if $\left\|A_{1}\right\|+\left\|A_{2}\right\|+\left\|A_{3}\right\|+$ $2\left\|A_{4}\right\|<1$. In this case, Corollary 7 holds true.

The following common fixed point theorem improves Theorem 2 of [23].

Theorem 8. Let $(X, p)$ be a $\theta$-complete partial cone metric space over a solid cone $P$ of a normed vector space $(E,\|\cdot\|)$ and $T, S: X \rightarrow X$. Assume that there exists $A \in \mathbb{Q}$ such that

$$
p(T x, S y) \preceq A p(x, y), \quad \forall x, y \in X .
$$

If $\rho(A)<1$, then $T$ and $S$ have a unique common fixed point $x^{*} \in X$ such that, for each $x_{0} \in X$, there exists some positive integer $n_{0}$ such that $x_{n} \stackrel{\tau_{p}}{\rightarrow} x^{*}$, where $x_{n}$ is defined by

$$
x_{n+1}= \begin{cases}T^{n_{0}} x_{n}, & n \text { is an even number }, \\ S^{n_{0}} x_{n}, & n \text { is an odd number. }\end{cases}
$$

Proof. By $\rho(A)<1$ and Gelfand's formula, there exists $0<$ $\beta<1$ such that $\lim _{n \rightarrow \infty} \sqrt[n]{\left\|A^{n}\right\|}=\rho(A) \leq \beta$, which implies that there exists a positive integer $n_{0}$ such that

$$
\left\|A^{n}\right\| \leq \beta^{n}, \quad \forall n \geq n_{0} .
$$

By (35), (36), and (p2),

$$
\begin{aligned}
p\left(x_{2 k+1}, x_{2 k+2}\right) & =p\left(T^{n_{0}} x_{2 k}, S^{n_{0}} x_{2 k+1}\right) \\
& \preceq A^{n_{0}} p\left(x_{2 k}, x_{2 k+1}\right), \\
p\left(x_{2 k+2}, x_{2 k+3}\right) & =p\left(T^{n_{0}} x_{2 k+2}, S^{n_{0}} x_{2 k+1}\right) \\
& \preceq A^{n_{0}} p\left(x_{2 k+1}, x_{2 k+2}\right), \quad \forall k \in \mathbb{N}
\end{aligned}
$$

and so

$$
p\left(x_{n}, x_{n+1}\right) \preceq A^{n_{0}} p\left(x_{n-1}, x_{n}\right), \quad \forall n \in \mathbb{N},
$$

which together with $A \in \mathfrak{Q}$ implies that

$$
p\left(x_{n}, x_{n+1}\right) \preceq A^{n n_{0}} p\left(x_{0}, x_{1}\right), \quad \forall n \in \mathbb{N} .
$$

Thus, by (p4),

$$
\begin{aligned}
p\left(x_{n}, x_{m}\right) & \preceq \sum_{i=n}^{m-1} p\left(x_{i}, y_{i+1}\right) \\
& \preceq \sum_{i=n}^{m-1} A^{i n_{0}} p\left(x_{0}, x_{1}\right), \quad \forall m>n .
\end{aligned}
$$

It follows from (37) that

$$
\begin{aligned}
& \left\|\sum_{i=n}^{m-1} A^{i n_{0}} p\left(x_{0}, x_{1}\right)\right\| \\
& \quad \leq\left\|p\left(x_{0}, x_{1}\right)\right\| \sum_{i=n}^{m-1}\left\|A^{n_{0}}\right\|^{i} \leq\left\|p\left(x_{0}, x_{1}\right)\right\| \sum_{i=n}^{m-1} \beta^{i n_{0}} \\
& \quad \leq \frac{\beta^{n n_{0}}\left\|p\left(x_{0}, x_{1}\right)\right\|}{1-\beta^{n_{0}}}, \quad \forall m>n,
\end{aligned}
$$

which implies $\sum_{i=n}^{m-1} A^{i n_{0}} p\left(x_{0}, x_{1}\right) \stackrel{\|\cdot\|}{\longrightarrow} \theta$, and hence $\sum_{i=n}^{m-1} A^{i n_{0}} p\left(x_{0}, x_{1}\right) \stackrel{w}{\longrightarrow} \theta$ by Lemma 2 . Therefore, by Lemma 1 and (41), we get $p\left(x_{n}, x_{m}\right) \stackrel{w}{\rightarrow} \theta$; that is, $\left\{x_{n}\right\}$ is a $\theta$-Cauchy sequence in $(X, p)$. Then, by analogy with the proof of Theorem 4, by $A \in \mathfrak{R}, \rho(A)<1$ and Lemma 3, we can prove that there exists some $x^{*} \in X$ with $p\left(x^{*}, x^{*}\right)=\theta$ such that $p\left(x_{n}, x^{*}\right) \stackrel{w}{\rightarrow} \theta$, and $x^{*}$ is the unique common fixed point of $T^{n_{0}}$ and $S^{n_{0}}$. For this $x^{*}$, we have $T^{n_{0}}\left(T x^{*}\right)=T\left(T^{n_{0}} x^{*}\right)=T x^{*}$ and $S^{n_{0}}\left(S x^{*}\right)=S\left(S^{n_{0}} x^{*}\right)=S x^{*}$; that is, $T x^{*}$ and $S x^{*}$ are fixed points of $T^{n_{0}}$ and $S^{n_{0}}$, respectively. It follows from (35) and $p\left(x^{*}, x^{*}\right)=\theta$ that $p\left(T x^{*}, S x^{*}\right) \preceq L p\left(x^{*}, x^{*}\right)=\theta$, and hence $T x^{*}=S x^{*}$ by (p1) and (p3). This shows that $T x^{*}$ is a common fixed point of $T^{n_{0}}$ and $S^{n_{0}}$. Note that $x^{*}$ is the unique common fixed point of $T^{n_{0}}$ and $S^{n_{0}}$; then, $T x^{*}=S x^{*}=x^{*}$; that is, $x^{*}$ is a common fixed point of $T$ and $S$. Moreover, it is easy to show that $x^{*}$ is the unique common fixed point of $T$ and $S$ by $A \in \mathfrak{Q}$ and $\rho(A)<1$. The proof is completed.

Example 9. Let $E=C_{\mathbb{R}}^{1}[0,1]$ with the norm $\|u\|=\|u\|_{\infty}+$ $\left\|u^{\prime}\right\|_{\infty}$, and $X=P=\{u \in E: u(t) \geq 0, t \in[0,1]\}$, which is 
nonnormal solid cone [24]. Define a mapping $p: X \times X \rightarrow P$ by

$$
p(x, y)= \begin{cases}x, & x=y \\ x+y, & \text { otherwise }\end{cases}
$$

It follows from Example 2 of [22] that $(X, p)$ is a partial cone metric space. Let $(A x)(t)=\int_{0}^{t} x(s) d s$ for each $x \in X$ and $t \in[0,1], T x=A x / 2$ and $S x=A x / 3$ for each $x \in X$. Clearly, $\theta$ is the unique common fixed point of $T$ and $S$.

By the definitions of $p, T, S$, and $A$,

$$
\begin{aligned}
& p(T x, S y) \\
& = \begin{cases}\theta=A p(x, y), & x=y=\theta, \\
\frac{5 A x}{6} \preceq A x=A p(x, y), & x=y \neq \theta, \\
\frac{A x}{2} \preceq \frac{5 A x}{2}=A p(x, y), & x \neq y, y=\frac{3 x}{2}, \\
\frac{A x}{2}+\frac{A y}{3} \preceq A x+A y=A p(x, y), & x \neq y, y \neq \frac{3 x}{2} ;\end{cases}
\end{aligned}
$$

that is, (35) is satisfied. It is clear that $\left(A^{n} x\right)(t) \leq\left(t^{n} / n !\right)\|x\|_{\infty}$ for each $t \in[0,1]$, and hence $\left\|A^{n} x\right\|_{\infty} \leq(1 / n !)\|x\|_{\infty}$. Note that $\left(A^{n} x\right)^{\prime}(t)=\left(A^{n-1} x\right)(t)$, and then

$$
\begin{aligned}
\left\|A^{n} x\right\| & =\left\|A^{n} x\right\|_{\infty}+\left\|\left(A^{n} x\right)^{\prime}\right\|_{\infty} \leq\left(\frac{1}{n !}+\frac{1}{(n-1) !}\right)\|x\|_{\infty} \\
& \leq\left(\frac{1}{n !}+\frac{1}{(n-1) !}\right)\|x\|,
\end{aligned}
$$

which implies that $\left\|A^{n}\right\| \leq\left(1^{n} / n !\right)+\left(1^{n} /(n-1) !\right)$. Therefore, by Gelfand's formula, $\rho(A)=\lim _{n \rightarrow \infty} \sqrt[n]{\left\|A^{n}\right\|}=0$ since $\lim _{n \rightarrow \infty}(1 / \sqrt[n]{n !})=0$, and hence $T$ and $S$ have a unique common fixed point by Theorem 8 .

Finally, we present a fixed point theorem of contractions restricted with positive linear bounded mappings, which generalizes Theorem 3.1 of [22].

Theorem 10. Let $(X, p)$ be a $\theta$-complete partial cone metric space over a solid cone $P$ of a normed vector space $(E,\|\cdot\|)$ and $T: X \rightarrow X$. Assume that there exist $A_{i} \in \mathfrak{L}(i=1,2,3,4,5)$ such that

$$
\begin{aligned}
p(T x, T y) \preceq & A_{1} p(x, y)+A_{2} p(x, T x) \\
& +A_{3} p(y, T y)+A_{4} p(x, T y) \\
& +A_{5} p(y, T x), \quad \forall x, y \in X .
\end{aligned}
$$

If $\left\|A_{2}+A_{4}\right\|<1$ and

$$
\left\|A_{2}+A_{3}+A_{4}+A_{5}\right\|+\left\|2 A_{1}+A_{2}+A_{3}+A_{4}+A_{5}\right\|<2,
$$

then $T$ and $S$ have a unique common fixed point $x^{*} \in X$ such that, for each $x_{0} \in X, x_{n} \stackrel{\tau_{p}}{\longrightarrow} x^{*}$, where $x_{n+1}=T x_{n}$ for all $n \in \mathbb{N}$.

Proof. Let $B=\left(A_{2}+A_{3}+A_{4}+A_{5}\right) / 2$. Then, $\|B\|<1$ by (47), and so the inverse of $B$ exists (denoted by $\left.(I-B)^{-1}\right)$. It follows from Neumann's formula that $(I-B)^{-1} \in \mathbb{Q}$ and

$$
\left\|(I-B)^{-1}\right\| \leq \frac{1}{1-\|B\|} .
$$

Let $K=(I-B)^{-1}\left(A_{1}+B\right)$. Then, $K \in \mathfrak{Q}$ by $(I-B)^{-1} \in \mathfrak{Q}$ and $A_{i} \in \mathfrak{Q}(i=1,2,3,4,5)$. Moreover, by (47) and (48),

$$
\|K\| \leq\left\|(I-B)^{-1}\right\|\left\|A_{1}+B\right\| \leq \frac{\left\|A_{1}+B\right\|}{1-\|B\|}<1 .
$$

By (46), (p4), and $A_{4} \in \mathbf{Q}$,

$$
\begin{aligned}
p\left(x_{n}, x_{n+1}\right)= & p\left(T x_{n-1}, T x_{n}\right) \\
\preceq & A_{1} p\left(x_{n-1}, x_{n}\right)+A_{2} p\left(x_{n-1}, x_{n}\right) \\
& +A_{3} p\left(x_{n}, x_{n+1}\right)+A_{4} p\left(x_{n-1}, x_{n+1}\right) \\
& +A_{5} p\left(x_{n}, x_{n}\right) \\
\preceq & A_{1} p\left(x_{n-1}, x_{n}\right)+A_{2} p\left(x_{n-1}, x_{n}\right) \\
& +A_{3} p\left(x_{n}, x_{n+1}\right) \\
& +A_{4}\left[p\left(x_{n-1}, x_{n}\right)+p\left(x_{n}, x_{n+1}\right)-p\left(x_{n}, x_{n}\right)\right] \\
& +A_{5} p\left(x_{n}, x_{n}\right), \quad \forall n \in \mathbb{N} .
\end{aligned}
$$

Similarly, take $x=x_{n}$ and $y=x_{n-1}$ in (46), and we get

$$
\begin{aligned}
p\left(x_{n+1}, x_{n}\right)= & p\left(T x_{n}, T x_{n-1}\right) \\
\leq & A_{1} p\left(x_{n-1}, x_{n}\right)+A_{2} p\left(x_{n}, x_{n+1}\right) \\
& +A_{3} p\left(x_{n-1}, x_{n}\right)+A_{4} p\left(x_{n}, x_{n}\right) \\
& +A_{5} p\left(x_{n-1}, x_{n+1}\right) \\
\leq & A_{1} p\left(x_{n-1}, x_{n}\right)+A_{2} p\left(x_{n}, x_{n+1}\right) \\
& +A_{3} p\left(x_{n-1}, x_{n}\right)+A_{4} p\left(x_{n}, x_{n}\right) \\
& +A_{5}\left[p\left(x_{n-1}, x_{n}\right)+p\left(x_{n}, x_{n+1}\right)\right. \\
& \left.-p\left(x_{n}, x_{n}\right)\right], \quad \forall n \in \mathbb{N} .
\end{aligned}
$$

It follows from (50) and (51) that

$$
(I-B) p\left(x_{n}, x_{n+1}\right) \preceq\left(A_{1}+B\right) p\left(x_{n-1}, x_{n}\right), \quad \forall n \in \mathbb{N} \text {. }
$$

Act the above inequality with $(I-B)^{-1}$; then, by $(I-B)^{-1} \epsilon$ \&,

$$
p\left(x_{n}, x_{n+1}\right) \preceq K p\left(x_{n-1}, x_{n}\right), \quad \forall n \in \mathbb{N},
$$


and so, by $K \in \mathfrak{Q}$,

$$
p\left(x_{n}, x_{n+1}\right) \preceq K^{n} p\left(x_{0}, x_{1}\right), \quad \forall n \in \mathbb{N} .
$$

By (p4),

$$
p\left(x_{n}, x_{m}\right) \leq \sum_{i=n}^{m-1} p\left(x_{i}, x_{i+1}\right) \leq \sum_{i=n}^{m-1} K^{i} p\left(x_{0}, x_{1}\right), \quad \forall m>n .
$$

It follows from (49) that

$$
\left\|\sum_{i=n}^{m-1} K^{i} p\left(x_{0}, x_{1}\right)\right\| \leq \frac{\|K\|^{n}\left\|p\left(x_{0}, x_{1}\right)\right\|}{1-\|K\|},
$$

which implies $\sum_{i=n}^{m-1} K^{i} p\left(x_{0}, x_{1}\right) \stackrel{\|\cdot\|}{\longrightarrow} \theta$, and hence $\sum_{i=n}^{m-1} K^{i} p\left(x_{0}, x_{1}\right) \stackrel{w}{\rightarrow} \theta$ by Lemma 2 . Therefore, by Lemma 1 and (55), $p\left(x_{n}, x_{m}\right) \stackrel{w}{\rightarrow} \theta$; that is, $\left\{x_{n}\right\}$ is a $\theta$-Cauchy sequence in $(X, p)$. By analogy with the proof of Theorem 4 , by $A_{i} \in \mathfrak{L}(i=1,2,3,4,5),\left\|A_{2}+A_{4}\right\|<1$, and Lemma 3, we can prove that there exists some $x^{*} \in X$ with $p\left(x^{*}, x^{*}\right)=\theta$ such that $p\left(x_{n}, x^{*}\right) \stackrel{w}{\rightarrow} \theta$ and $x^{*}$ is a fixed point of $T$. Note that (47) implies $\left\|A_{1}+A_{2}+A_{3}+A_{4}+A_{5}\right\|<1$; then, similar to the proof of Theorem 4 , we can show $x^{*}$ is the unique fixed point of $T$. The proof is completed.

Remark 11. It is easy to check that all the conditions of Theorem 10 are satisfied if $\sum_{i=1}^{5}\left\|A_{i}\right\|<1$. Therefore, Theorem 10 is valid with $\sum_{i=1}^{n}\left\|A_{i}\right\|<1$, and hence Theorem 3.1 of [22] is a special case of Theorem 10 with $A_{i}=c_{i} I(i=$ $1,2,3,4,5)$, where $c_{i}(i=1,2,3,4,5)$ are four nonnegative real numbers such that $\sum_{i=1}^{5} c_{i}<1$.

\section{Conflict of Interests}

The authors declare that there is no conflict of interests regarding the publication of this paper.

\section{Acknowledgments}

The work was supported by the Natural Science Foundation of China (11161022), Natural Science Foundation of Jiangxi Province (20114BAB211006, 20122BAB201015), Educational Department of Jiangxi Province (GJJ12280, GJJ13297), and Program for Excellent Youth Talents of JXUFE (201201).

\section{References}

[1] L.-G. Huang and X. Zhang, "Cone metric spaces and fixed point theorems of contractive mappings," Journal of Mathematical Analysis and Applications, vol. 332, no. 2, pp. 1468-1476, 2007.

[2] L. Kantorovitch, "The method of successive approximations for functional equations," Acta Mathematica, vol. 71, pp. 63-97, 1939.

[3] L. V. Kantorovič, "The principle of the majorant and Newton's method," Doklady Akademii Nauk SSSR, vol. 76, pp. 17-20, 1951.

[4] L. V. Kantorovitch, "On some further applications of the Newton approximation method," Vestnik Leningradskogo Universiteta, vol. 12, no. 7, pp. 68-103, 1957.
[5] W. A. Kirk and B. C. Kang, "A fixed point theorem revisited," Journal of the Korean Mathematical Society, vol. 34, pp. 285-291, 1972.

[6] K. J. Chung, "Nonlinear contractions in abstract spaces," Kodai Mathematical Journal, vol. 4, no. 2, pp. 288-292, 1981.

[7] M. A. Krasnoseljski and P. P. Zabreiko, Geometrical Methods in Nonlinear Analysis, Springer, 1984.

[8] I. A. Rus, A. Petrusel, and G. Petrusel, Fixed Point Theory, Cluj University Press, Cluj-Napoca, Romania, 2008.

[9] R. P. Agarwal, "Contraction and approximate contraction with an application to multipoint boundary value problems," Journal of Computational and Applied Mathematics, vol. 9, no. 4, pp. 315-325, 1983.

[10] N. Cakić, Z. Kadelburg, S. Radenović, and A. Razani, "Common fixed point results in cone metric spaces for a family of weakly compatible maps," Advances and Applications in Mathematical Sciences, vol. 1, no. 1, pp. 183-207, 2009.

[11] S. Janković, Z. Kadelburg, S. Radenović, and B. E. Rhoades, "Assad-Kirk-type fixed point theorems for a pair of nonself mappings on cone metric spaces," Fixed Point Theory and Applications, vol. 2009, article 16, Article ID 761086, 2009.

[12] G. Jungck, S. Radenović, S. Radojević, and V. Rakočević, "Common fixed point theorems for weakly compatible pairs on cone metric spaces," Fixed Point Theory and Applications, vol. 2009, article 13, Article ID 643840, 2009.

[13] Z. Kadelburg, S. Radenović, and V. Rakočević, "Remarks on "Quasi-contraction on a cone metric space',' Applied Mathematics Letters, vol. 22, no. 11, pp. 1674-1679, 2009.

[14] Z. Kadelburg, S. Radenović, and B. Rosić, "Strict contractive conditions and common fixed point theorems in cone metric spaces," Fixed Point Theory and Applications, vol. 2009, article 14, Article ID 173838, 2009.

[15] S. Radenović and B. E. Rhoades, "Fixed point theorem for two non-self mappings in cone metric spaces," Computers \& Mathematics with Applications, vol. 57, no. 10, pp. 1701-1707, 2009.

[16] S. Janković, Z. Kadelburg, and S. Radenović, "On cone metric spaces: a survey," Nonlinear Analysis: Theory, Methods \& Applications, vol. 74, no. 7, pp. 2591-2601, 2011.

[17] W.-S. Du, "A note on cone metric fixed point theory and its equivalence," Nonlinear Analysis: Theory, Methods \& Applications, vol. 72, no. 5, pp. 2259-2261, 2010.

[18] W.-S. Du, "Nonlinear contractive conditions for coupled cone fixed point theorems," Fixed Point Theory and Applications, vol. 2010, article 16, Article ID 190606, 2010.

[19] S. G. Matthews, "Partial metric topology," in Proceedings of the 8th Summer Conference on General Topology and Applications, vol. 728, pp. 183-197, Academy of Sciences, New York, NY, USA, 1994.

[20] A. Sonmez, "Fixed point theorems in partial cone metric spaces, Arxiv," 2011, http://arxiv.org/abs/1101.2741vl.

[21] A. Sonmez, "On partial cone metric space, Arxiv," 2012, http:// arxiv.org/abs/arXiv:1207.6766vl.

[22] S. K. Malhotra, S. Shukla, and R. Sen, "Some fixed point results in $\theta$-complete partial cone metric spaces," Journal of Advanced Mathematical Studies, vol. 6, no. 2, pp. 97-108, 2013.

[23] S. Jiang and Z. Li, "Extensions of Banach contraction principle to partial cone metric spaces over a non-normal solid cone," Fixed Point Theory and Applications, vol. 2013, article 250, 2013.

[24] K. Deimling, Nonlinear Functional Analysis, Springer, Berlin, Germany, 1985. 


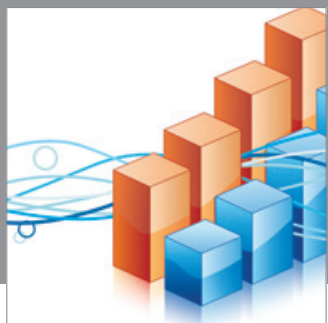

Advances in

Operations Research

mansans

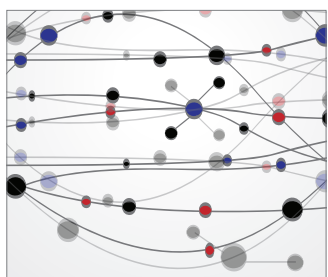

The Scientific World Journal
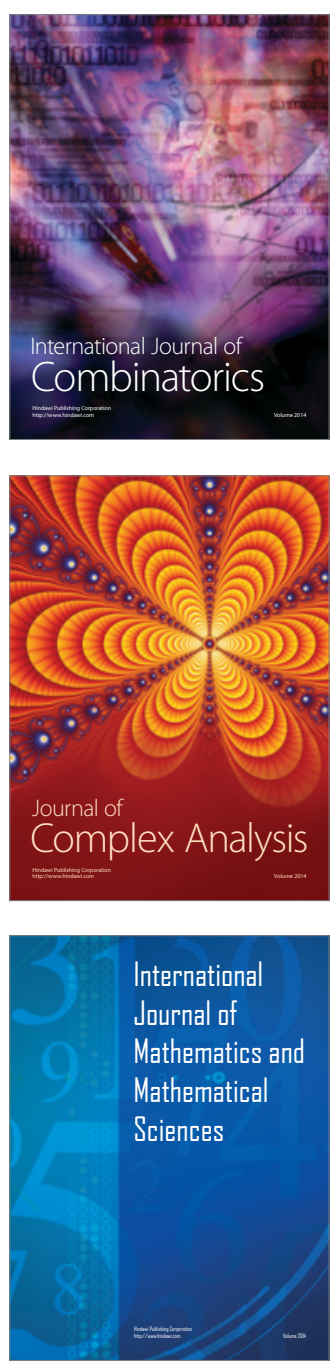
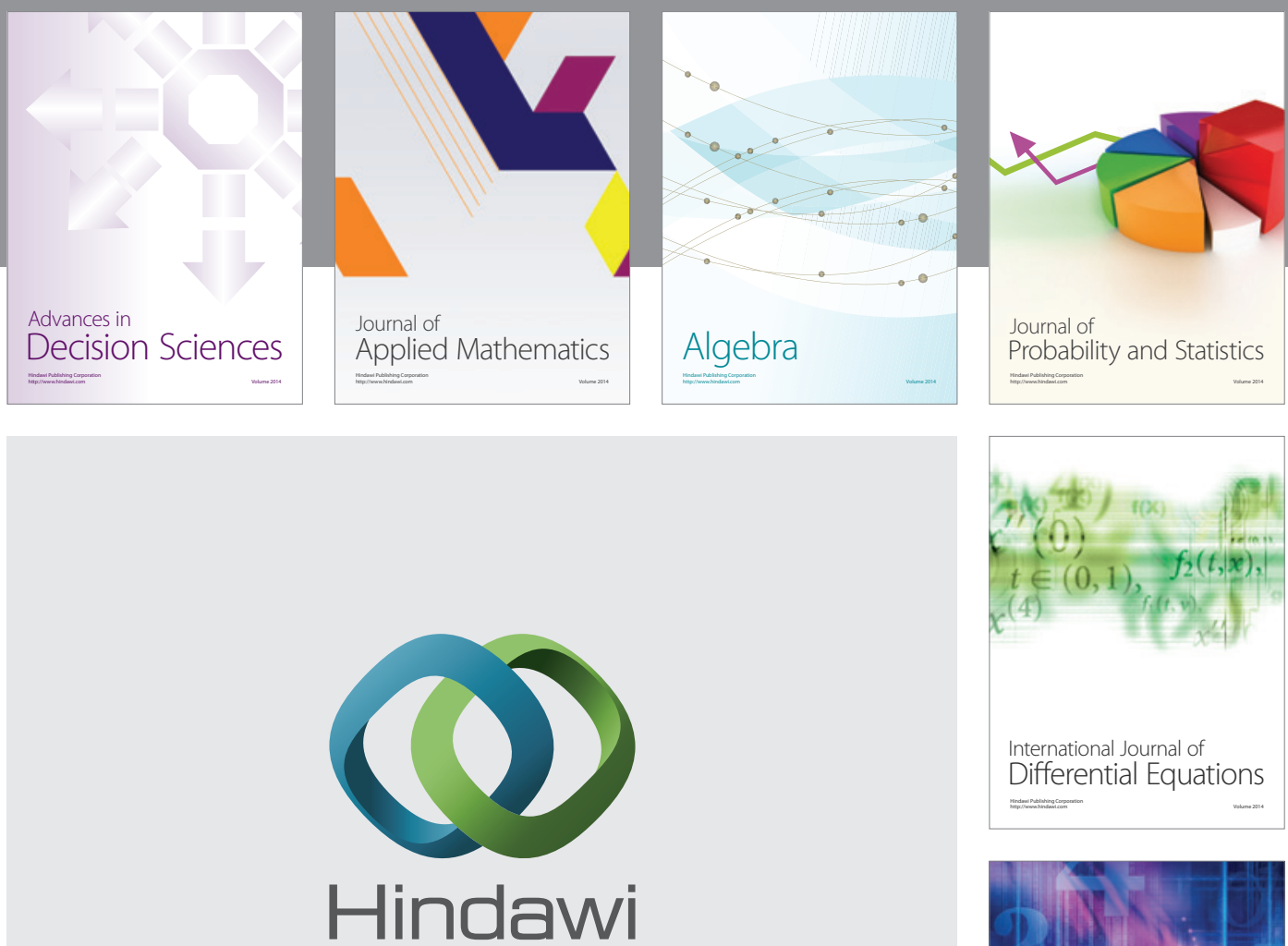

Submit your manuscripts at http://www.hindawi.com
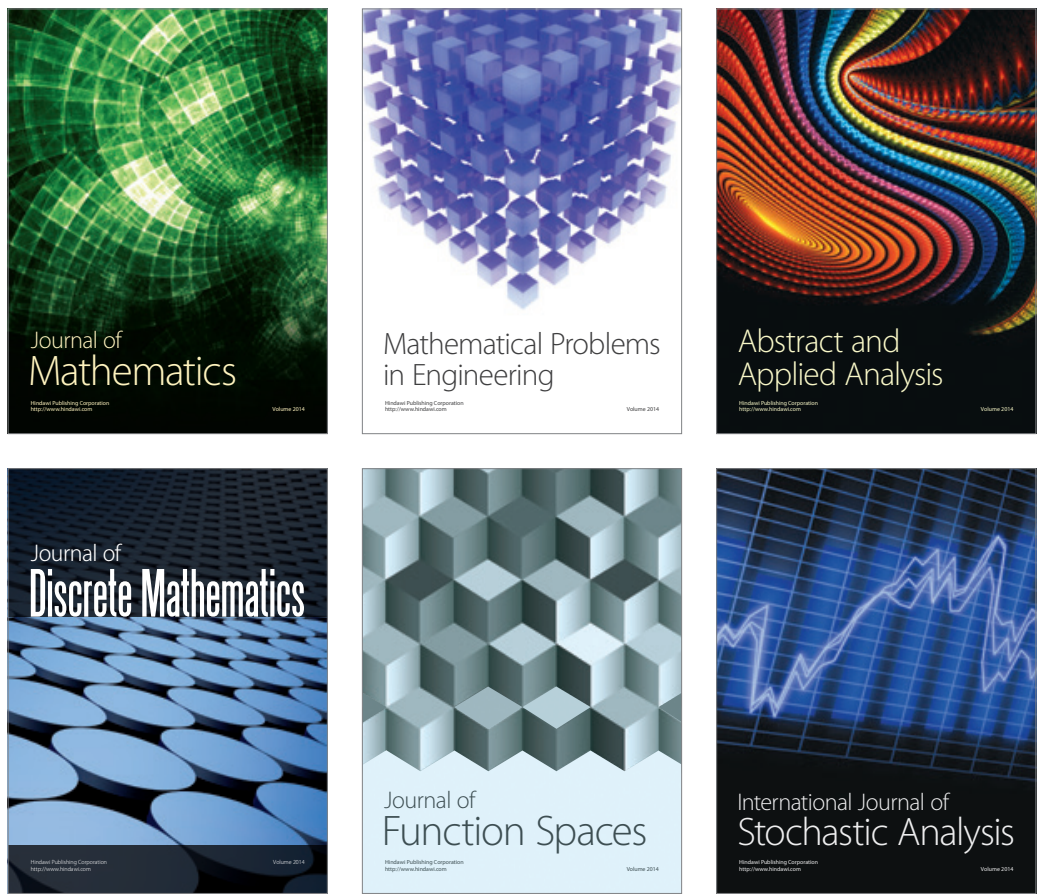

Journal of

Function Spaces

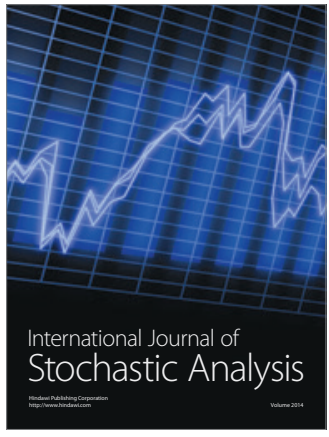

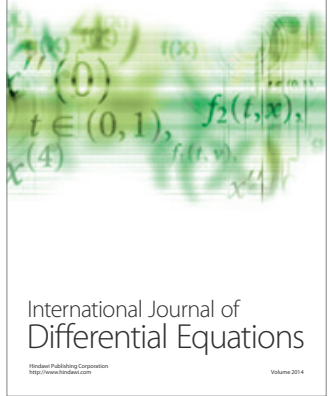
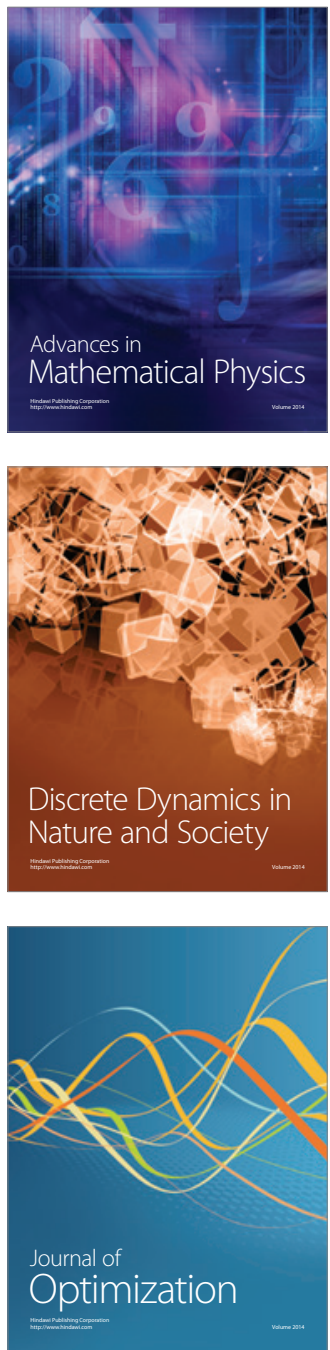